\title{
Complex Microbiome in Brain Abscess Revealed by Whole-Genome Culture-Independent and Culture-Based Sequencing
}

\author{
Jyun-Hong Lin ${ }^{1,+}$, Zong-Yen $\mathrm{Wu}^{2,+}{ }^{+}$, Liang Gong ${ }^{3}$, Chee-Hong Wong ${ }^{3}$, Wen-Cheng Chao ${ }^{4}$, \\ Chun-Ming Yen ${ }^{5,6}$, Ching-Ping Wang ${ }^{7}$, Chia-Lin Wei ${ }^{3, *}$, Yao-Ting Huang ${ }^{1, *}$ and \\ Po-Yu Liu $5,8, *$ (D) \\ 1 Department of Computer Science and Information Engineering, National Chung Cheng University, \\ Chia-Yi 62102, Taiwan; twolinin@gmail.com \\ 2 Department of Veterinary Medicine, National Chung Hsing University, Taichung 40227, Taiwan; \\ zongyen@gmail.com \\ 3 Genome Technologies, The Jackson Laboratory for Genomic Medicine, Farmington, CT 06032, USA; \\ Liang.Gong@jax.org (L.G.); CheeHong.Wong@jax.org (C.-H.W.) \\ 4 Department of Medical Research, Taichung Veterans General Hospital, Taichung 40705, Taiwan; \\ cwc081@hotmail.com \\ 5 Program in Translational Medicine, National Chung Hsing University, Taichung 40227, Taiwan; \\ chunmingyen@gmail.com \\ 6 Department of Neurosurgery, Neurological Institute, Taichung Veterans General Hospital, \\ Taichung 40705, Taiwan \\ 7 Department of Otolaryngology-Head and Neck Surgery, Taichung Veterans General Hospital, \\ Taichung 40705, Taiwan; entcpw@gmail.com \\ 8 Division of Infectious Diseases, Department of Internal Medicine, Taichung Veterans General Hospital, \\ Taichung 40705, Taiwan \\ * Correspondence: Chia-lin.Wei@jax.org (C.-L.W.); ythuang@cs.ccu.edu.tw (Y.-T.H.); \\ pyliu@vghtc.gov.tw (P.-Y.L.) \\ + These authors contributed equally to this work.
}

Received: 6 February 2019; Accepted: 7 March 2019; Published: 12 March 2019

\begin{abstract}
Brain abscess is a severe infectious disease with high mortality and mobility. Although culture-based techniques have been widely used for the investigation of microbial composition of brain abscess, these approaches are inherent biased. Recent studies using $16 \mathrm{~S}$ ribosomal sequencing approaches revealed high complexity of the bacterial community involved in brain abscess but fail to detect fungal and viral composition. In the study, both culture-independent nanopore metagenomic sequencing and culture-based whole-genome sequencing using both the Illumina and the Nanopore platforms were conducted to investigate the microbial composition and genomic characterization in brain abscess. Culture-independent metagenomic sequencing revealed not only a larger taxonomic diversity of bacteria but also the presence of fungi and virus communities. The culture-based whole-genome sequencing identified a novel species in Prevotella and reconstructs a Streptococcus constellatus with a high GC-skew genome. Antibiotic-resistance genes $C f x A$ and ErmF associated with resistance to penicillin and clindamycin were also identified in culture-based and culture-free sequencing. This study implies current understanding of brain abscess need to consider the broader diversity of microorganisms.
\end{abstract}

Keywords: metagenomics; whole genome sequencing; genomics; brain abscess; Streptococcus constellatus; Prevotella 


\section{Introduction}

Brain abscess is one of the most life-threatening infectious diseases, commonly resulting from contiguous spread from an adjacent infected focus [1]. Despite diagnostic and therapeutic advances, the mortality remains high-from 15 to $85 \%$ [1]. The reported causative organisms vary depending on the clinical circumstances. Brain abscess may be caused by bacteria, fungi and parasites [1]. In most cases, the detection of causative organisms is made by culture of drainage abscess. However, culture-negative brain abscess is not uncommon, occurring in between $9 \%$ and $63 \%$ of patients in difference series [2-5]. Hence, the microbial spectrum involved in brain abscess is incompletely characterized.

The applications of advanced sequencing technology enable culture-independent approaches for the detection of difficult-to-culture or unculturable taxa. There are several studies that have been conducted using $16 \mathrm{~S}$ rRNA gene amplicon sequencing to characterize bacterial community in brain abscess [6-9]. While these studies have detected microbial signatures of brain abscess, they are based on the amplification of a specific gene of bacteria, leaving other microbial community (i.e., fungi and virus) largely unexplored [10]. For instance, fungi have also been identified in brain abscesses, showing that investigation of microbiome needs to extend beyond the typical bacterial 16S rRNA gene sequencing.

The development of culture-independent whole-genome metagenomic sequencing offers an unbiased approach for investigating the entire microbial community as well as the genetic factors in the genomes [11]. The whole-genome metagenomic approach to clinical specimens randomly samples DNA en masse. In contrast, targeted approaches using specific amplification are biased to the detection of a subpopulation and a small subset of genetic elements [12].

The present study applied both culture-independent and culture-based sequencing for comprehensively characterizing brain abscess microbial communities. Complete genomes of cultured isolates were reconstructed and analyzed using a combination of Illumina and MinION Nanopore sequencing. Culture-independent metagenomes were sequenced by Nanopore platform, disclosing a larger diversity of microorganisms including bacteria, fungi and virus.

\section{Materials and Methods}

\subsection{The Case}

A 60-year-old man presented with fever and headache. He had a history of adenoid cystic carcinoma of left maxillary sinus and received left total maxillectomy, orbital exenteration, external ethmoidectomy and sphenoidectomy. The MRI revealed heterogeneous enhancement with focal rim enhancing cystic lesion surrounding with perifocal edema, indicating brain abscess (Figure 1). This brain abscess specimen was collected by stereotaxic aspiration. The patient consented the aspirates sent for conventional microbiology survey and metagenomic analyses. This research was approved by the Institutional Review Board of Taichung Veterans General Hospital (CE16111B).

\subsection{Conventional Bacterial Strain Identification and Susceptibility Test}

Aspirate from the abscess was subjected to culture. The specimens was inoculated onto Trypticase-soy agar supplemented with 5\% horse blood (bioMérieux, Marcy l'Etoile, France), chocolate agar plate (bioMérieux, Marcy l'Etoile, France) and eosin-methylene blue agar plate (Becton Dickinson, Sparks, MD, USA). $\pm 1 \mathrm{~mL}$ of the sample was inoculated in thioglycolate broth (bioMérieux, Marcy l'Etoile, France) for enrichment. Agar plates and broth were incubated aerobically at $37^{\circ} \mathrm{C}$. For anaerobic culture, CDC anaerobe agar plate with $5 \%$ Sheep Blood (Becton Dickinson, Sparks, MD, USA) were used. The plate was incubated fat $37^{\circ} \mathrm{C}$ in anaerobic condition. Preliminary identification of isolates were performed using the Vitek 2 system (bioMérieux, Marcy l'Etoile, France) and the Sanger sequencing of 16S rRNA amplified by PCR using universal primers 27F(5'-AGAGTTTGATCCTGGCTCAG-3') and 1492R(5'-TACGGYTACCTTGTTACGACTT3'). 
16S rRNA gene-based identification reported Streptococcus sp. (Designated TCV107) and Prevotella sp. (Designated TCVGH). The in vitro susceptibility of the isolates were determined by the Vitek 2 system (bioMérieux, Marcy l’Etoile, France).

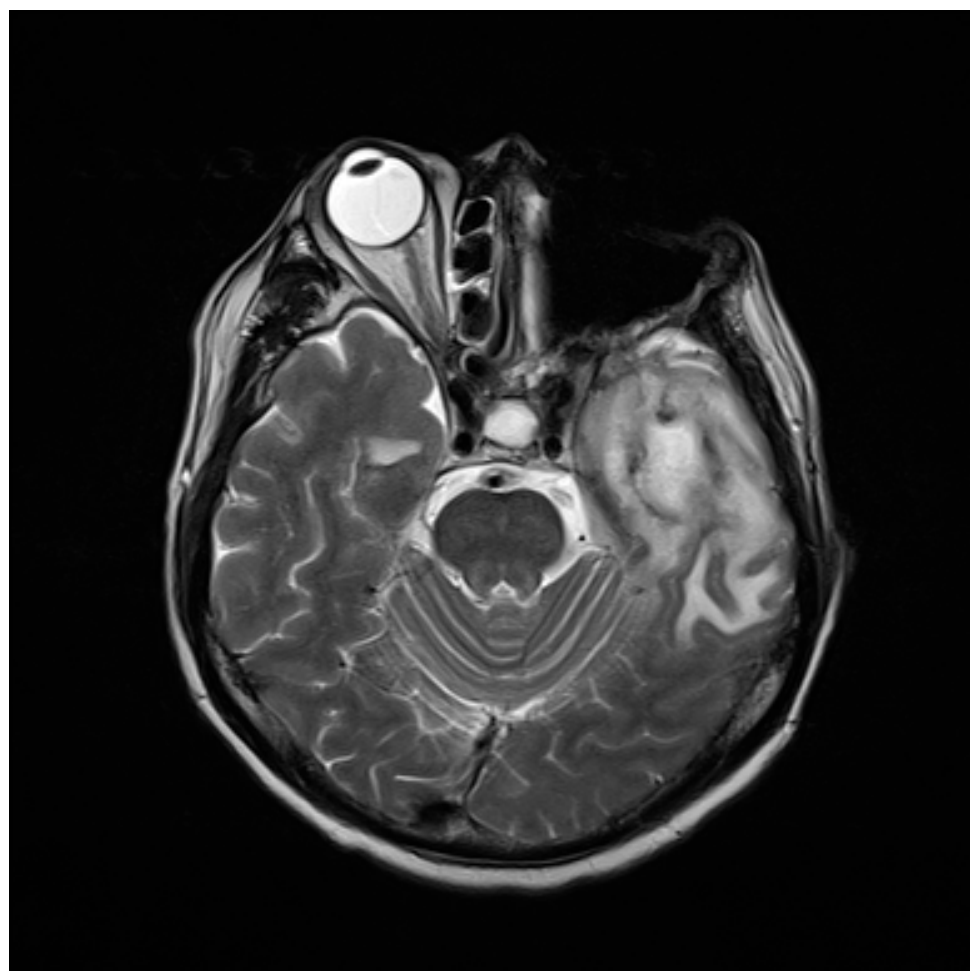

Figure 1. Magnetic resonance image (MRI) of brain showing heterogeneous enhancement with focal rim enhancing cystic lesions in left anterior temporal lobe.

\subsection{DNA Extraction}

Genomic DNA of Streptococcus constellatus TCV107 and Prevotella sp. TCVGH was prepared from overnight liquid cultures grown in MAS (Medium for Acinetobacter Supplemented) broth at $30^{\circ} \mathrm{C}$ with shaking to an O.D.600 of approximately 1.5. Cells were pelleted and lysed in the presence of Lysozyme from chicken egg white (Sigma, St. Louis, MO, USA). Genomic DNA was purified by phenol-chloroform (Sigma) phase extraction. Extracted DNA was resolved in $100 \mu \mathrm{L}$ TE buffer $(10 \mathrm{mM}$ Tris, $1 \mathrm{mM}$ EDTA [pH 8.0]) supplemented with $10 \mu \mathrm{g} / \mathrm{mL}$ RNase (Sigma).

\subsection{Illumina Library Preparation and Sequencing of Isolates from Brain Abscess}

DNA (30-100 ng) was sonicated to a 100-800 bp size range using a Covaris E210 sonicator (Covaris, Woburn, MA, USA). Fragments were end-repaired, 3'-adenylated and Illumina adapters were then added using the NEBNext Sample Reagent Set (New England Biolabs, Ipswich, MA, USA). Ligation products were purified using Ampure XP (Beckmann Coulter Genomics, Danvers, MA, USA) and DNA fragments (>200 bp) were PCR amplified using Illumina adapter-specific primers and Platinum Pfx DNA polymerase (Invitrogen, Carlsbad, CA, USA). Amplified library fragments of 650-750 bp were size selected on a 3\% agarose gel. Libraries were quantified by qPCR using the KAPA Library Quantification Kit for Illumina Libraries (KapaBiosystems, Wilmington, MA, USA) and library profiles were assessed using a DNA High Sensitivity LabChip kit on an Agilent Bioanalyzer (Agilent Technologies, Santa Clara, CA, USA). Libraries were sequenced on an Illumina MiSeq instrument (San Diego, CA, USA) using 300 base-length read chemistry in a paired-end mode. 


\subsection{Nanopore Library Preparation and Sequencing of Isolates from Brain Abscess}

Library preparation was performed using the 1D Genomic DNA sequencing kit SQK-LSK108 (Oxford Nanopore Technologies) with the omission of DNA shearing and DNA repair steps to prevent further DNA fragmentation. Library preparation was initiated at the DNA end-prep step. All cleanup steps were performed using AMPure XP beads (Beckman Coulter). The final $80 \mu \mathrm{L}$ prepared library was proceeded to sequencing on the MinION Mk1b device using a FLO-MIN-106 R9.4 flow cell (Oxford Nanopore Technologies, Oxford, UK) using the MinKNOW software for the full $48 \mathrm{~h}$ run time with no alterations to any voltage scripts.

\subsection{Metagenomic Sequencing}

Aspirated abscess was diluted in $1 \mathrm{~mL} 0.9 \%$ sodium chloride. The sample was sedimented by centrifugation at $500 \mathrm{~g}$ for $5 \mathrm{~min}$ at $4{ }^{\circ} \mathrm{C}$. The supernatant was centrifuged again at $800 \mathrm{~g}$ for $5 \mathrm{~min}$ at $4{ }^{\circ} \mathrm{C}$ for the separation of the human cells. DNA was extracted with standard silica mini-columns (Qiagen Genomic-tip 20/G) following the manufacturer's instruction. DNA purity and concentration were determined using Nanodrop (NanoDrop 2000, Thermo Fisher Scientific, Waltham, MA, USA). Approximately 400-500 ng DNA was taken to construct a DNA library for nanopore sequencing using a Rapid Sequencing Kit (SQK-RAD003 from Oxford Nanopore Technologies, Oxford, UK) as described by the manufacturer and then loaded onto a MinION Mk1b device using a FLO-MIN-106 R9.4 flow cell (Oxford Nanopore Technologies, Oxford, UK) following the standard 48-h run scripts.

\subsection{Genome Assembly and Gene Annotation}

The TCV107 and TCVGH isolates were sequenced by both Nanopore and Illumina platform. The sequences were assembled using Canu v1.5 [13] and SPAdes v3.11.1 [14] software. The assembled genome were further polished using Racon v1.3.1 [15] followed by Nanopolish v0.9.0 [16]. Finally, the polished genome was circularized using Circlator v1.5.5 [17]. Species identification were conducted via MIGA and BLAST scan of NCBI microbiome database, indicating presence of Streptococcus constellatus TCV107 and Prevotella sp. TCVGH. Gene annotation was performed via National Center for Biotechnology Information (NCBI) Prokaryotic Genomes Automatic Annotation Pipeline (PGAAP). The TCVGH and TCV107 genomes as well as annotations have been deposited in NCBI with accession numbers QFFX00000000 and CP029207. GC skew was calculated using a 10kb window sliding along the entire genome for calculating $(C-G) /(C+G)$ ratio. In order to determine the loci of ori and ter in TCV107, we extracted ori and ter sequences from Streptococcus pyogene M3 genome (ori: 1,650 kb-230 kb, ter: $\sim 920-~ 1100 \mathrm{~kb}$ ) and mapped them onto the Streptococcus constellatus TCV107 genome by BLAST.

\subsection{Taxonomic Classification}

The de novo assembled genomes indicated novel species (e.g., Prevotella sp. TCVGH) not presented in existing NCBI microbiome database. As the sequences are a mixture of human and unknown microbiome, we construct a more comprehensive database by integrating the human genome, NCBI microbiome database and de novo assembled genomes from culture-based sequencing. Minimap2 was used to align culture-free ONT reads against the new integrated database. Because short reads lack specificity during classification, reads with a length less than $500 \mathrm{bp}$ were discarded. In addition, reads with insufficient alignment coverage $(<70 \%$ of the original read length are also filtered. The top hits of the remaining read alignments were extracted to plot the taxonomic classification. Both cutoffs were determined by statistics of read length and coverage (Supplementary Figure S1). 


\subsection{Identification of Antimicrobial-Resistant Genes and Virulence Factors}

The Comprehensive Antibiotic Resistance Database (CARD) [18] and the virulence factor database [19] were used for annotation of virulence and antimicrobial factors. The method was further optimized separately for culture-based and culture-free sequencing. In the culture-free sequencing, the resistome of TCV107 and TCVGH were annotated by using the Resistance Gene Identifier (RGI) from CARD. RGI prediction of resistome is based on homology and SNP models. In homolog models, BLAST is used to detect functional homologs of antimicrobial resistance genes. In contrast, SNP models identify candidate genes which acquire mutations conferring antimicrobial resistance genes based on curated SNP matrices. The virulence factors were predicted through BLAST alignments against the VFDB database using a 95\% sequence identity threshold. In the antibiotic-resistance genes annotation of culture-free sequencing, ONT reads were mapped onto antibiotic-resistance genes in CARD by BLASTX. Due to the high error rate of ONT reads, we observed a lot of false-positive alignments. In order to achieve higher specificity than sensitivity, the threshold of alignment identity is set to $100 \%$ and e-value less than 1.

\section{Results}

The cultured isolates were sequenced using Nanopore and Illumina platforms and culture-free metagenomic sequencing of brain abscess sample was performed using Nanopore platform (Figure 2, Supplementary Tables S1 and S2). Culture-based sequencing reconstructed entire bacterial genomes from isolates, which provided genomic features and insight into taxonomy, virulence and antibiotic-resistant determinants. On the other hand, culture-free sequencing is essential to unbiasedly reveal complex microbial communities, in particular with regard to uncultivated organisms.

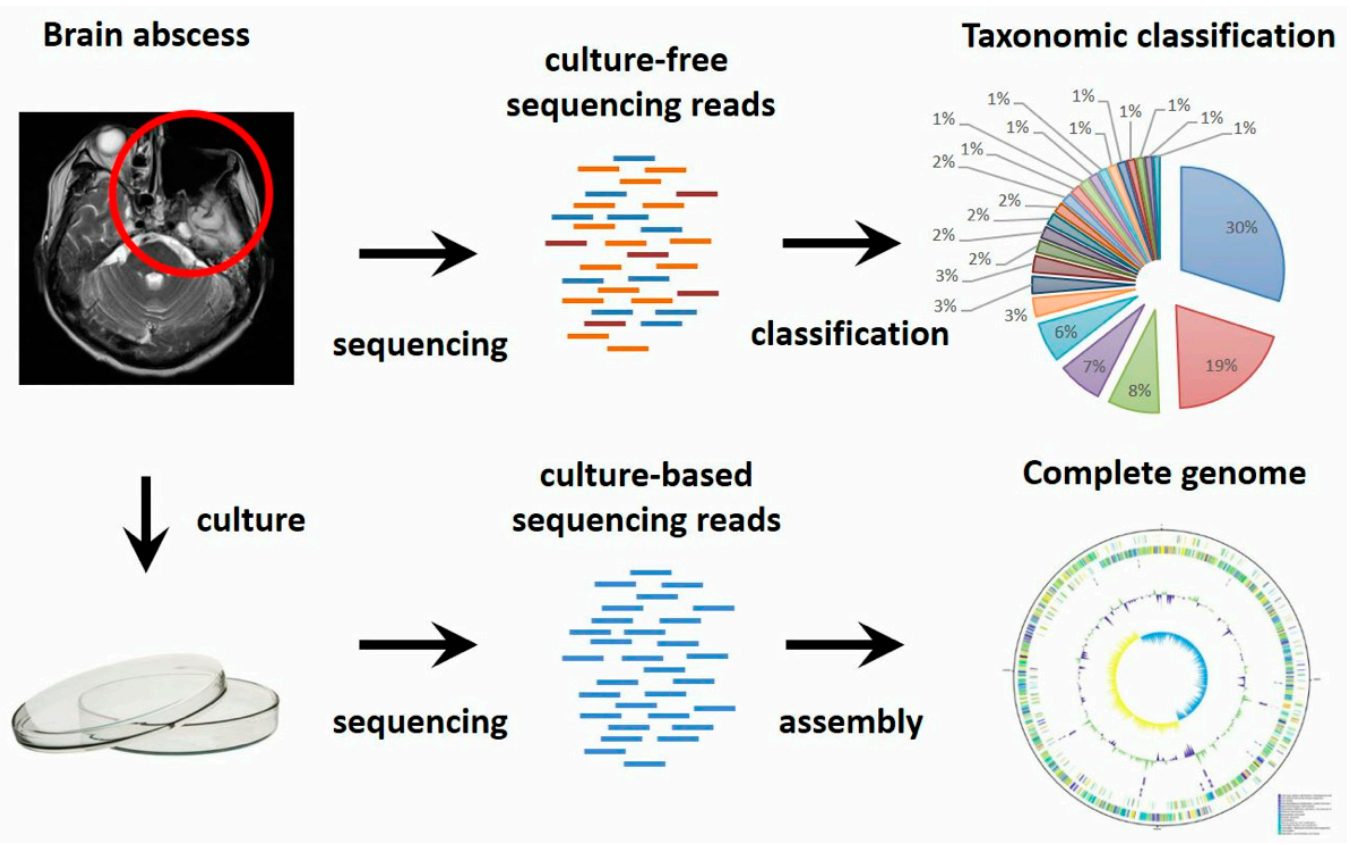

Figure 2. Experimental workflow of culture-based and culture-free sequencing. Brain abscess was first confirmed by MRI. The abscess sample was cultivated and followed by sequencing and assembly. The sample was also directly sequenced in order to disclose the taxonomic composition of entire community including uncultivated bacteria.

\subsection{Novel Bacterial Genome Revealed by Culture-Based Sequencing}

Through hybrid sequencing and de novo assembly of cultured isolates, we obtained two whole genomes: a 3.06Mb Prevotella sp. TCVGH and a 1.96Mb Streptococcus constellatus TCV107 (Figure 3 and Table 1). The TCVGH genome is most similar to Prevotella enoeca genome, yet only $70.29 \%$ average 
nucleotide identity (ANI) value (Figure 3a, Supplementary Figure S2). Consequently, it was classified as a novel Prevotella subspecies (Prevotella sp.) under the Prevotellacea family $(p=0.0018)$ and likely belonging to the Prevotella genus $(p=0.31)$. The complete genome of TCV107 was unambiguously classified as Streptococcus constellatus (with $97.03 \%$ ANI). The TCV107 genome exhibits prominent GC-skews (Figure 3b). The two skew breakpoints contain the replication origin (ori) and terminus (ter) sequences, which divides the genome into two halves possibly corresponding to replichores. The replication leading and lagging strands are dominant with nucleotide $G$ and with $C$, respectively. In addition, protein-coding genes are largely presented on the replication leading strand and usually absent on the lagging strand. Functional annotation of the TCVGH and TCV107 genomes revealed 2569 and 2008 protein-coding genes, respectively. Gene functions were grouped into 25 categories according to Clusters of Orthologous Groups (COGs) (Supplementary Tables S3 and S4). Replication, recombination and repair genes are the major category in TCVGH genome, while genes involving carbohydrate transport and metabolism are the dominant class in TCV107 genome.

a

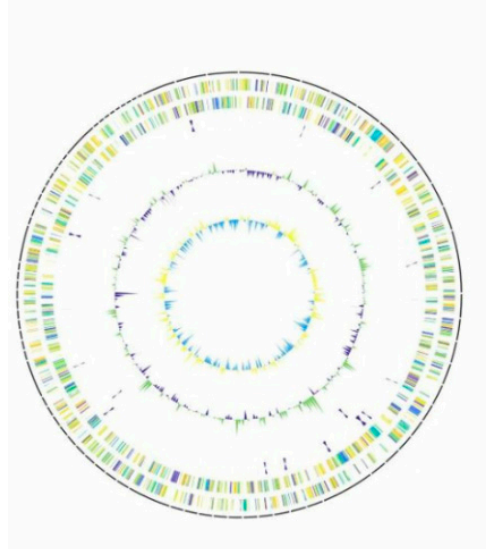

b

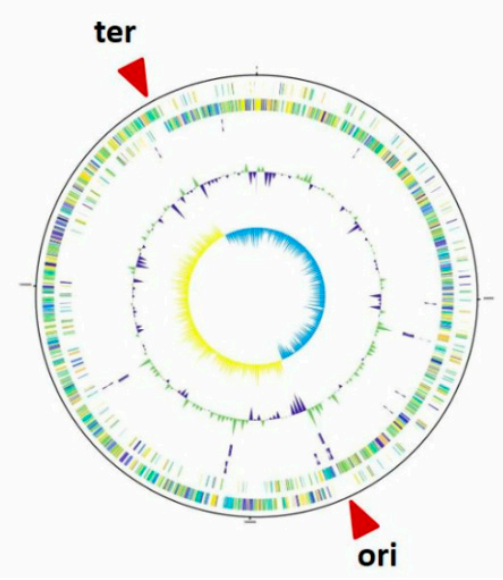

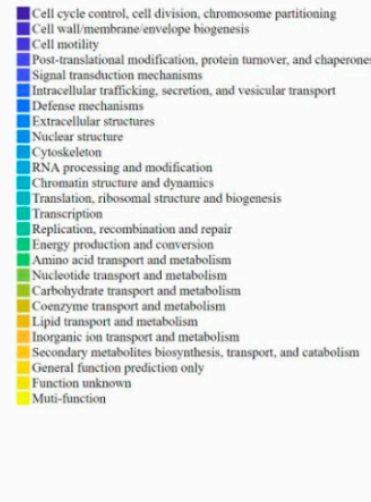

Figure 3. Circular genome map of Prevotella sp. TCVGH (a) and Streptococcus constellatus TCV107 (b). Circular genome maps. From outer to inner circles: genes on forward strand, genes on reverse strand, rRNA, GC content and GC skew. (a) Circular genome map of Prevotella sp. TCVGH; (b) Circular genome map of Streptococcus constellatus TCV107. The innermost circle shows strong and opposite GC skews in the leading and lagging strands, partitioning the genome into two halves separated by replication origin (ori) and terminus (ter) sequences. The two outer circles exhibit strong gene orientation bias, whereas half genes are dominantly on the forward strand and the other half are on the reverse strand, partitioned by ter and ori.

Table 1. Genomic data of Prevotella sp. TCVGH and Streptococcus constellatus TCV107.

\begin{tabular}{cccccc}
\hline Species & $\begin{array}{c}\text { Genome Size } \\
(\mathbf{b} \mathbf{p})\end{array}$ & $\begin{array}{c}\text { GC Content } \\
\mathbf{( \% )}\end{array}$ & $\begin{array}{c}\text { Genes (Pseudo } \\
\text { Genes) }\end{array}$ & CDSs & tRNAs \\
\hline Prevotella sp. TCVGH & $3,061,518$ & 42.6 & $2569(110)$ & 2409 & 43 \\
Streptococcus constellatus TCV107 & $1,954,689$ & 38.11 & $2008(125)$ & 1809 & 59 \\
\hline
\end{tabular}

\subsection{Complex Communities of Bacteria, Fungi and Virus Revealed by Culture-Independent Metagenomic} Sequencing

Culture-free metagenomic sequencing of the brain abscess sample disclosed a complex microbial community. Using strict read-mapping and taxonomic-classification criteria (see Method), we recovered $\sim 36 \%$ of microbiome reads from the metagenomic sequencing, whereas human sequences occupied $29.6 \%$ and the remaining reads were unmapped to neither human genome nor any genome in NCBI microbiome database. Taxonomic classification of microbiome reads revealed not only a larger bacterial community but also the communities of fungi and virus (Figure 4). 


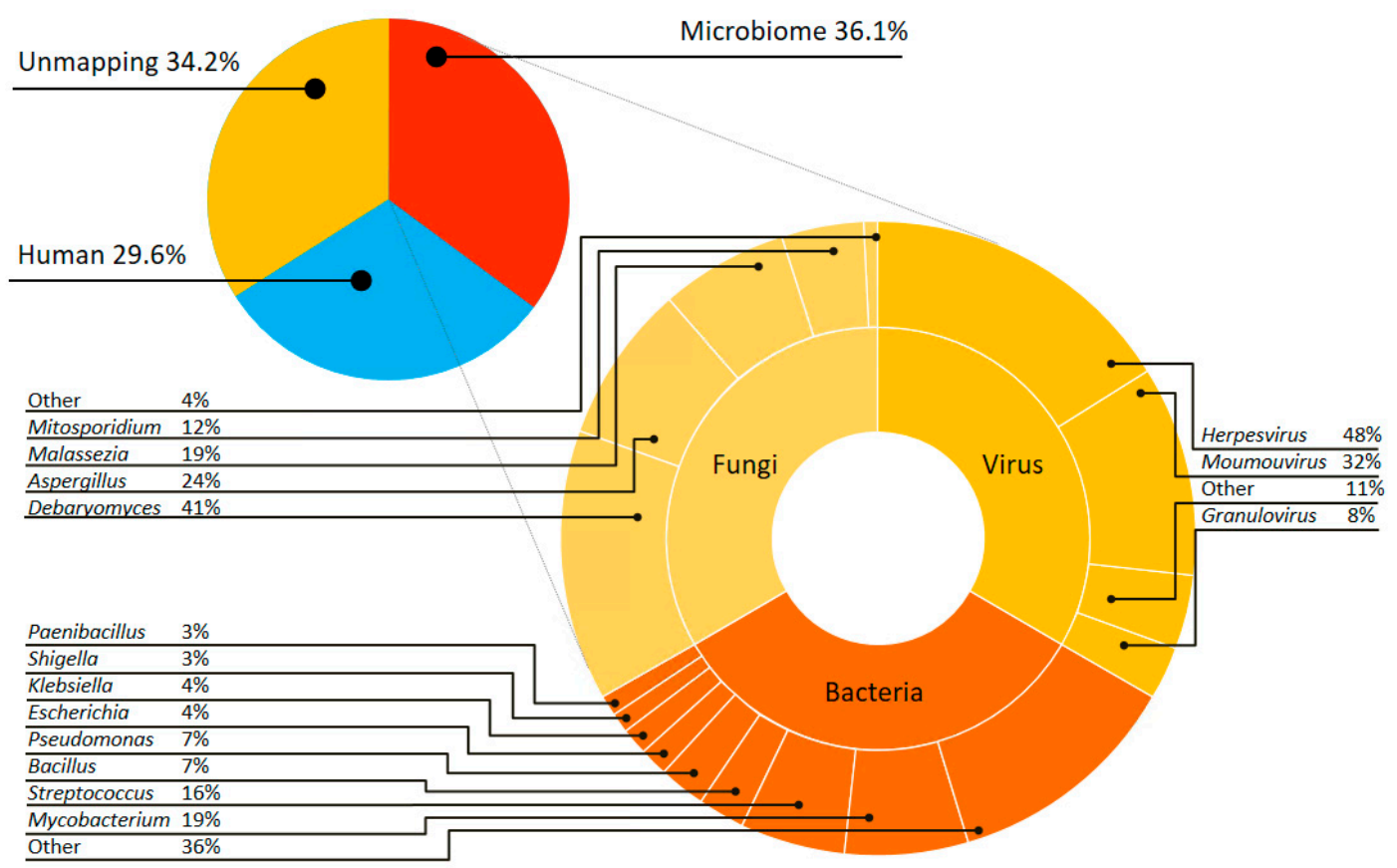

Figure 4. Microbial composition of brain abscesses. Taxonomic classification of culture-free sequencing. In total, microbiome occupies $36.1 \%$ while human contamination and unmapping reads takes $29.6 \%$ and $34.2 \%$, respectively. The microbiome reads are further classified into bacteria $(97.5 \%)$, fungi $(1.5 \%)$ and virus $(1 \%)$ communities. The species within each community are shown in the outer circle.

The bacterial community is composed of more than ten genera (Supplementary Table S5). Although the two cultured isolates (i.e., Streptococcus constellatus TCV107 and Prevotella sp. TCVGH) were successfully re-discovered, the most-frequent bacteria is Mycobacterium ( 19\%), followed by Streptococcus $(\sim 16 \%)$ and the assembled Prevotella genome only takes $\sim 0.24 \%$ in the community. The fungi community is dominant by Debaryomyces (41\%), followed by Aspergillus (24\%), Malassezia $(19 \%)$ and Mitosporidiym (12\%) (Supplementary Table S6). The virus community consists of Herpesvirus (48\%), Moumouvirus (32\%) and Granulovirus (8\%) (Supplementary Table S7). To our best knowledge, this is the first report of the existence of both fungi and virus communities in brain abscess, implying different clinical treatments may be required and the need for culture-free sequencing.

\subsection{Antibiotic Resistance Genes Revealed by Culture-Based and Culture-Independent Metagenomic Sequencing}

Streptococcus constellatus TCV107 was susceptible to all tested antibiotics, including penicillin, vancomycin, clindamycin and erythromycin, whereas Prevotella sp. TCVGH was susceptible to metronidazole, cefoxitin and chloromycin but resistant to penicillin and clindamycin (Supplementary Tables S8 and S9). By using antibiotic-resistant database (CARD) and virulence factor database (VFDB), we identified four antibiotic-resistant genes (ErmF, tet $Q, C f x A 3, C f x A 2)$ in TCVGH genome and two virulence factors (has $C$ and $p s a A$ ) in TCV107 genome (Table 2, Supplementary Table S10). The antibiotic-resistance genes are all encoded on the TCVGH chromosome, of them $C f x A 2 / C f x A 3$ and $E r m F$ are known penicillin- and chloromycin-resistant determinants, respectively. Finally, we assess the sensitivity of antibiotic-resistance genes detection in culture-free sequencing of the same abscess sample, which is mixed with human cells. While missing CfxA and ErmF, tet $Q$ was successfully rediscovered in the metagenomic sample (Supplementary Figure S1). 
Table 2. Predicted resistant genes in Prvotella sp. TCVGH.

\begin{tabular}{ccccc}
\hline Gene & Coverage (\%) & Identity (\%) & $\begin{array}{c}\text { Detected in } \\
\text { Isolated Genome }\end{array}$ & $\begin{array}{c}\text { Detected in } \\
\text { Metagenomics }\end{array}$ \\
\hline ErmF & 100 & 99.38 & $y$ & \\
$t e t Q$ & 99.34 & 97.91 & $y$ & $y$ \\
CfxA3 & 100 & 100 & $y$ & \\
CfxA2 & 100 & 99.9 & $y$ & \\
\hline
\end{tabular}

\section{Discussion}

The study reveals a highly diverse microbial population in brain abscess. Our findings are complementary to those of previous studies for bacteria $[6,20]$. The dominant reads of Bacteria domain were related to Streptococcus, Pseudomonas and Escherichia (Supplementary Table S6). Streptococcus is a common pathogen in head and neck infections and has been associated with brain abscess caused by contiguous spread from ear or paranasal sinus [21]. Pseudomonas and Escherichia have been reported in brain abscess related to surgical procedure and paranasal sinus infection [22]. The bacterial spectrum is consistent with the host factors and reflects the polymicrobial nature of brain abscess resulting from parameningeal foci of the head and neck and previous surgical procedures.

Among the Fungi domain, the three most dominant genus were Debaryomyces, Aspergillus and Malassezia. The association of Debaryomyces with central nervous system infection has been reported [23]. Aspergillus and Malassezia are difficult-to-culture commensal of skin and known pathogens of brain abscess [24,25]. In this sense, the association of Aspergillus and Malassezia with brain abscess could be related to damage of the natural protective barriers [24]. Herpesvirus is the most dominant virus type in the study and one of the most common viral causes of encephalitis [26].

Depending on the employed method, the detected microbial communities were significantly different. Although culture-based techniques have been widely used for the investigation of microbial composition of brain abscess, these approaches are inherently biased by the media utilized for growth, result in limited data available on the spectrum of causative organisms. 16S rDNA-based targeted sequencing revealed unculturable bacteria but cannot identify fungus and virus. Shotgun metagenomics provides opportunities explore a microbial community with a wide context.

In Streptococcus constellatus TCV107 the two regions of GC skew divide the genome into two halves and the two breakpoints contain the replication origin (ori) and terminus (ter) sequences, which possibly corresponding to two replichores. This implies the GC skew is likely correlated with DNA replication [27]. Furthermore, protein-coding genes are also partitioned into two halves by ori and ter, whereas one half is largely presented on the forward strand, while the other half are mostly on the reverse strand. Strong nucleotide composition bias and gene-orientation bias were previously found in intracellular parasites [28].

Of the four antibiotic-resistance genes found in TCVGH genome (i.e., $C f x A 2, C f x A 3, E r m F$, tet $Q$ ), $C f x A 2$ and $C f x A 3$, are likely the main factors leading to penicillin resistance [29]. In addition, ErmF is a known chloromycin-resistant determinant, which can alter the binding site of clindamycin via encoding an erythromycin resistance methylase [30]. tetQ often accompanies with ErmF and associated with reduced susceptibility to tetracycline in other Prevotella species.

The large fraction of unmapped reads were also reported in References [31-33] and partly due to the incompleteness of existing database biased against uncultivated microorganisms [34]. Though not emphasized in the paper, we ever assembled the metagenomic reads in the hope of reconstructing uncultivated microorganisms. But due to insufficient DNA from clinical sample, only five small contigs (two human and three plasmids) were assembled. Hence, taxonomic classification of this study was still mainly based on existing microbiome database, which may miss uncultivated microorganisms. Another limitation is the possible contamination. Contamination from reagents and device do impact sequence-based microbiome analyses [35], in particular for sample types that have low microbial 
biomass [36]. Some experts suggest concurrent sequencing of negative control samples [35] and strict laboratory condition to reduce the impact of contaminants [37].

\section{Conclusions}

In summary, we showed that metagenomic sequencing was concordant with the conventional culture-based method. Moreover, the unbiased nature of metagenomic DNA sequencing allowed an expanded scope of pathogen detection including bacteria, fungal species and viruses, while concurrently providing drug resistance information. These data suggest a practical diagnostic decision tree whereby samples negative by routine culture are then advanced to both metagenomic sequencing. This approach will not only complement the current diagnostic paradigm but also allow for a more comprehensive characterization of the etiology of brain abscess.

Supplementary Materials: The following are available online at http:/ /www.mdpi.com/2077-0383/8/3/351/ s1, Figure S1: Taxonomic classification of metagenomic data using partial alignment ratio and read length, Figure S2: Average nucleotide identity analysis of Prevotella sp. TCVGH, Table S1: Sequencing data from Illumina MiSeq, Table S2: MinIon Nanopore sequencing data, Table S3: COG functional CDS of Prevotella sp. TCVGH, Table S4: COG functional CDS of Streptococcus constellatus TCV107, Table S5: Metagenome analysis of microbial composition, Table S6: Metagenome analysis of fungal composition, Table S7: Metagenome analysis of viral composition, Table S8: Antimicrobial susceptibility testing of Streptococcus constellatus TCV107, Table S9: Antimicrobial susceptibility testing of Prevotella sp. TCVGH, Table S10: Predicted virulence gene in Streptococcus constellatus TCV107, Table S11: Comparison of metagenomic analysis in the study with previous study.

Author Contributions: Conceptualization, C.-L.W., Y.-T.H. and P.-Y.L.; Formal analysis, Z.-Y.W., Y.-T.H. and P.-Y.L.; Funding acquisition, P.-Y.L.; Investigation, W.-C.C., C.-M.Y., C.-P.W. and C.-L.W.; Methodology, Z.-Y.W., L.G. and C.-H.W.; Project administration, Z.-Y.W.; Resources, L.G. and C.-L.W.; Software, J.-H.L., C.-H.W. and Y.-T.H.; Supervision, C.-L.W.; Validation, J.-H.L., C.-L.W. and Y.-T.H.; Visualization, J.-H.L.; Writing-Original draft, J.-H.L., Y.-T.H. and P.-Y.L.; Writing-Review \& editing, L.G. and C.-L.W.

Funding: This research was funded by Taichung Veterans General Hospital, grant number TCVGH-1083901B. YTH was supported in part by the Ministry of Science and Technology (MOST) with grant numbers 103-2923-E-194-001-MY3 and 104-2221-E-194-048-MY2.

Acknowledgments: I would like to take this opportunity to thank Wayne Huey-Herng Sheu. This work would not have been possible without his encouragement and support. The authors would also like to thank Hirofumi Sawa, Michihito Sasaki, Manabu Igarashi and Junya Yamagishi from Hokkaido University Research Center for Zoonosis Control for sharing the experience of next-generation sequencing. We would also like to take this opportunity to thank Lee Pan for his support in Kyoto and Osaka.

Conflicts of Interest: The authors declare no conflict of interest.

\section{References}

1. Brouwer, M.C.; Tunkel, A.R.; McKhann, G.M., 2nd; van de Beek, D. Brain abscess. N. Engl. J. Med. 2014, 371, 447-456. [CrossRef]

2. De Louvois, J.; Gortavai, P.; Hurley, R. Bacteriology of abscesses of the central nervous system: A multicentre prospective study. Br. Med. J. 1977, 2, 981-984. [CrossRef] [PubMed]

3. Prasad, K.N.; Mishra, A.M.; Gupta, D.; Husain, N.; Husain, M.; Gupta, R.K. Analysis of microbial etiology and mortality in patients with brain abscess. J. Infect. 2006, 53, 221-227. [CrossRef]

4. Tseng, J.H.; Tseng, M.Y. Brain abscess in 142 patients: Factors influencing outcome and mortality. Surg. Neurol. 2006, 65, 557-562, discussion 562. [CrossRef] [PubMed]

5. Roche, M.; Humphreys, H.; Smyth, E.; Phillips, J.; Cunney, R.; McNamara, E.; O’Brien, D.; McArdle, O. A twelve-year review of central nervous system bacterial abscesses; presentation and aetiology. Clin. Microbiol. Infect. 2003, 9, 803-809. [CrossRef] [PubMed]

6. Al Masalma, M.; Lonjon, M.; Richet, H.; Dufour, H.; Roche, P.H.; Drancourt, M.; Raoult, D.; Fournier, P.E. Metagenomic analysis of brain abscesses identifies specific bacterial associations. Clin. Infect. Dis. 2012, 54, 202-210. [CrossRef]

7. Tsai, J.C.; Teng, L.J.; Hsueh, P.R. Direct detection of bacterial pathogens in brain abscesses by polymerase chain reaction amplification and sequencing of partial 16s ribosomal deoxyribonucleic acid fragments. Neurosurgery 2004, 55, 1154-1162. [CrossRef] 
8. Al Masalma, M.; Armougom, F.; Scheld, W.M.; Dufour, H.; Roche, P.H.; Drancourt, M.; Raoult, D. The expansion of the microbiological spectrum of brain abscesses with use of multiple 16s ribosomal DNA sequencing. Clin. Infect. Dis. 2009, 48, 1169-1178. [CrossRef] [PubMed]

9. Mishra, A.K.; Dufour, H.; Roche, P.H.; Lonjon, M.; Raoult, D.; Fournier, P.E. Molecular revolution in the diagnosis of microbial brain abscesses. Eur. J. Clin. Microbiol. Infect. Dis. 2014, 33, 2083-2093. [CrossRef]

10. Gong, L.; Huang, Y.T.; Wong, C.H.; Chao, W.C.; Wu, Z.Y.; Wei, C.L.; Liu, P.Y. Culture-independent analysis of liver abscess using nanopore sequencing. PLoS ONE 2018, 13, e0190853. [CrossRef] [PubMed]

11. Guo, L.Y.; Feng, W.Y.; Guo, X.; Liu, B.; Liu, G.; Dong, J. The advantages of next-generation sequencing technology in the detection of different sources of abscess. J. Infect. 2019, 78, 75-86. [CrossRef] [PubMed]

12. Cheng, J.; Hu, H.; Kang, Y.; Chen, W.; Fang, W.; Wang, K.; Zhang, Q.; Fu, A.; Zhou, S.; Cheng, C.; et al. Identification of pathogens in culture-negative infective endocarditis cases by metagenomic analysis. Ann. Clin. Microbiol. Antimicrob. 2018, 17, 43. [CrossRef] [PubMed]

13. Koren, S.; Walenz, B.P.; Berlin, K.; Miller, J.R.; Bergman, N.H.; Phillippy, A.M. Canu: Scalable and accurate long-read assembly via adaptive k-mer weighting and repeat separation. Genome Res. 2017, 27, 722-736. [CrossRef] [PubMed]

14. Bankevich, A.; Nurk, S.; Antipov, D.; Gurevich, A.A.; Dvorkin, M.; Kulikov, A.S.; Lesin, V.M.; Nikolenko, S.I.; Pham, S.; Prjibelski, A.D.; et al. Spades: A new genome assembly algorithm and its applications to single-cell sequencing. J. Comput. Biol. 2012, 19, 455-477. [CrossRef] [PubMed]

15. Vaser, R.; Sovic, I.; Nagarajan, N.; Sikic, M. Fast and accurate de novo genome assembly from long uncorrected reads. Genome Res. 2017, 27, 737-746. [CrossRef] [PubMed]

16. Loman, N.J.; Quick, J.; Simpson, J.T. A complete bacterial genome assembled de novo using only nanopore sequencing data. Nat. Methods 2015, 12, 733-735. [CrossRef] [PubMed]

17. Hunt, M.; Silva, N.D.; Otto, T.D.; Parkhill, J.; Keane, J.A.; Harris, S.R. Circlator: Automated circularization of genome assemblies using long sequencing reads. Genome Biol. 2015, 16, 294. [CrossRef] [PubMed]

18. Jia, B.; Raphenya, A.R.; Alcock, B.; Waglechner, N.; Guo, P.; Tsang, K.K.; Lago, B.A.; Dave, B.M.; Pereira, S.; Sharma, A.N.; et al. Card 2017: Expansion and model-centric curation of the comprehensive antibiotic resistance database. Nucleic Acids Res. 2017, 45, D566-D573. [CrossRef]

19. Liu, B.; Zheng, D.; Jin, Q.; Chen, L.; Yang, J. Vfdb 2019: A comparative pathogenomic platform with an interactive web interface. Nucleic Acids Res. 2019, 47, D687-D692. [CrossRef]

20. Hu, H.L.; Guo, L.Y.; Wu, H.L.; Feng, W.Y.; Chen, T.M.; Liu, G. Evaluation of next-generation sequencing for the pathogenic diagnosis of children brain abscesses. J. Infect. 2019. [CrossRef]

21. Brouwer, M.C.; Coutinho, J.M.; van de Beek, D. Clinical characteristics and outcome of brain abscess: Systematic review and meta-analysis. Neurology 2014, 82, 806-813. [CrossRef] [PubMed]

22. Patel, K.; Clifford, D.B. Bacterial brain abscess. Neurohospitalist 2014, 4, 196-204. [CrossRef] [PubMed]

23. Alonso, R.; Pisa, D.; Marina, A.I.; Morato, E.; Rabano, A.; Rodal, I.; Carrasco, L. Evidence for fungal infection in cerebrospinal fluid and brain tissue from patients with amyotrophic lateral sclerosis. Int. J. Biol. Sci. 2015, 11, 546-558. [CrossRef] [PubMed]

24. Ashbee, H.R. Update on the genus malassezia. Med. Mycol 2007, 45, 287-303. [CrossRef] [PubMed]

25. Bhaskar, N.; Mohammad, K.; Kaur, V. Aspergillus brain abscess. J. Ark. Med. Soc. 2013, 110, 41-42.

26. Tyler, K.L. Acute viral encephalitis. N. Engl. J. Med. 2018, 379, 557-566. [CrossRef] [PubMed]

27. Arakawa, K.; Tomita, M. Measures of compositional strand bias related to replication machinery and its applications. Curr. Genom. 2012, 13, 4-15. [CrossRef] [PubMed]

28. Zhao, H.L.; Xia, Z.K.; Zhang, F.Z.; Ye, Y.N.; Guo, F.B. Multiple factors drive replicating strand composition bias in bacterial genomes. Int. J. Mol. Sci. 2015, 16, 23111-23126. [CrossRef] [PubMed]

29. Sherrard, L.J.; Schaible, B.; Graham, K.A.; McGrath, S.J.; McIlreavey, L.; Hatch, J.; Wolfgang, M.C.; Muhlebach, M.S.; Gilpin, D.F.; Schneiders, T.; et al. Mechanisms of reduced susceptibility and genotypic prediction of antibiotic resistance in prevotella isolated from cystic fibrosis (cf) and non-cf patients. J. Antimicrob. Chemother. 2014, 69, 2690-2698. [CrossRef]

30. Lofmark, S.; Jernberg, C.; Jansson, J.K.; Edlund, C. Clindamycin-induced enrichment and long-term persistence of resistant bacteroides spp. And resistance genes. J. Antimicrob. Chemother. 2006, 58, 1160-1167. [CrossRef] 
31. Greninger, A.L.; Naccache, S.N.; Federman, S.; Yu, G.; Mbala, P.; Bres, V.; Stryke, D.; Bouquet, J.; Somasekar, S.; Linnen, J.M.; et al. Rapid metagenomic identification of viral pathogens in clinical samples by real-time nanopore sequencing analysis. Genome Med. 2015, 7, 99. [CrossRef] [PubMed]

32. Batovska, J.; Lynch, S.E.; Rodoni, B.C.; Sawbridge, T.I.; Cogan, N.O. Metagenomic arbovirus detection using MinION nanopore sequencing. J. Virol. Methods 2017, 249, 79-84. [CrossRef] [PubMed]

33. Anderson, R.E.; Sogin, M.L.; Baross, J.A. Evolutionary Strategies of Viruses, Bacteria and Archaea in Hydrothermal Vent Ecosystems Revealed through Metagenomics. PLoS ONE 2014, 9, e109696. [CrossRef] [PubMed]

34. Parks, D.H.; Rinke, C.; Chuvochina, M.; Chaumeil, P.A.; Woodcroft, B.J.; Evans, P.N.; Hugenholtz, P.; Tyson, G.W. Recovery of nearly 8,000 metagenome-assembled genomes substantially expands the tree of life. Nat. Microbiol. 2017, 2, 1533-1542. [CrossRef] [PubMed]

35. Salter, S.J.; Cox, M.J.; Turek, E.M.; Calus, S.T.; Cookson, W.O.; Moffatt, M.F.; Turner, P.; Parkhill, J.; Loman, N.J.; Walker, A.W. Reagent and laboratory contamination can critically impact sequence-based microbiome analyses. BMC Biol. 2014, 12, 87. [CrossRef]

36. Eisenhofer, R.; Minich, J.J.; Marotz, C.; Cooper, A.; Knight, R.; Weyrich, L.S. Contamination in low microbial biomass microbiome studies: Issues and recommendations. Trends Microbiol. 2019, 27, 105-117. [CrossRef]

37. Greninger, A.L.; Messacar, K.; Dunnebacke, T.; Naccache, S.N.; Federman, S.; Bouquet, J.; Mirsky, D.; Nomura, Y.; Yagi, S.; Glaser, C.; et al. Clinical metagenomic identification of balamuthia mandrillaris encephalitis and assembly of the draft genome: The continuing case for reference genome sequencing. Genome Med. 2015, 7, 113. [CrossRef]

(C) 2019 by the authors. Licensee MDPI, Basel, Switzerland. This article is an open access article distributed under the terms and conditions of the Creative Commons Attribution (CC BY) license (http:/ / creativecommons.org/licenses/by/4.0/). 